\title{
Erratum to: Validity Issues of Digital Trace Data for Platform as a Service: A Network Science Perspective
}

Mehmet N. Aydin, Dzordana Kariniauskaite, and N. Ziya Perdahci

\section{Erratum to:}

Chapter "Validity Issues of Digital Trace Date for Platform as a Service: A Network Science Perspective" in: Á. Rocha et al. (Eds.): Trends and Advances in Information Systems and Technologies, AISC 745, https://doi.org/10.1007/978-3-319-77703-0_65

In the original version of the book, the misspelt second author name "Dzordana Kariniasukaite" has been corrected to read as "Dzordana Kariniauskaite" in Chapter 65 , frontmatter and backmatter, and the term "Date" in the title of Chapter 65 has been replaced with "Data" so that it should read as "Validity Issues of Digital Trace Data for Platform as a Service: A Network Science Perspective". 